\title{
Reconstruction of Boundary Controls in Parabolic Systems
}

\author{
A. I. Korotkii ${ }^{1,2}$ and D. O. Mikhailova ${ }^{1}$ \\ Received April 27, 2011
}

\begin{abstract}
In the paper, an inverse dynamic problem is considered. It consists in reconstructing a priori unknown boundary controls in dynamical systems described by boundary value problems for partial differential equations of parabolic type. The source information for solving the inverse problem is the results of approximate measurements of the states of the observed system's motion. The problem is solved in the static case; i.e., to solve it, we use all the measurement data accumulated during some specified observation interval. The problem under consideration is ill-posed. To solve it, we propose the Tikhonov method with a stabilizer containing the sum of the mean-square norm and total time variation of the control. The use of such nondifferentiable stabilizer allows us to obtain more precise results than the approximation of the desired control in the Lebesgue spaces. In particular, this method provides the pointwise and piecewise uniform convergences of regularized approximations and makes possible the numerical reconstruction of the subtle structure of the desired control. In the paper, the subgradient projection method for obtaining a minimizing sequence for the Tikhonov functional is described and substantiated. Also, we demonstrate the two-stage finitedimensional approximation of the problem and present the results of numerical simulation.

Keywords: dynamical system, control, reconstruction, observation, measurement, inverse problem, regularization, the Tikhonov method, variation, piecewise uniform convergence.
\end{abstract}

DOI: $10.1134 / \mathrm{S} 0081543813020090$

\section{INTRODUCTION}

In this paper, we consider the problem of reconstructing a priori unknown boundary controls acting in controlled dynamical systems described by boundary value problems for partial differential equations of parabolic type. Control actions in a dynamical system can be a priori unknown and must be determined as a result of observing the system from approximate measurements of its current phase states. Similar reconstruction problems for dynamical systems were studied in different statements in control theory, in the theory of differential games, and in the theory of estimation and identification [1-9]. It is well known that the problems under consideration are ill-posed and their solution requires the application of regularization methods [10-12].

To solve the problem, the Tikhonov variational method consisting in the minimization of some appropriate residual functional over the set of admissible controls is used. The problem is solved in

\footnotetext{
${ }^{1}$ Institute of Mathematics and Mechanics, Ural Branch of the Russian Academy of Sciences, ul. S. Kovalevskoi 16, Yekaterinburg, 620990 Russia

emails: korotkii@imm.uran.ru,darso@rambler.ru

${ }^{2}$ Institute of Mathematics and Computer Science, Ural Federal University, ul. Mira, 19, Yekaterinburg, 620000 Russia
} 
a static case; i.e., to solve it, we use all the measurement data accumulated during some specified interval of observing the system. The emphasis is on designing regularizing algorithms, which are able to reconstruct the desired control not only in the traditional mean-square metric but also in stronger metrics.

It is known that, for ill-posed problems, the classical Tikhonov regularization, which uses the norms of Sobolev spaces as stabilizers, provides a high reconstruction quality for smooth desired functions but does not allow to qualitatively reconstruct nondifferentiable functions, which may have breakpoints, close peaks, discontinuities, and other singularities. Controls acting in dynamical systems may possess such features. Stabilizing functionals containing the norms of Sobolev spaces have a strong regularizing effect, which necessarily results in smoothing a desired function and losing its subtle structure. Stabilizing functionals containing the norms of Lebesgue spaces are often used for regularizing problems but they also result in a rather rough approximation. Therefore, there arises the necessity to construct stabilizers that are specially designed for reconstructing nonsmooth functions and functions with singularities. Within variational regularization methods, several classes of stabilizing functionals have been suggested so far and proved to be rather efficient in the reconstruction of both smooth and nonsmooth functions. In the case of functions of one variable, stabilizers containing the classical or generalized variation combined with some strictly convex norm are often used [13-23]. On this path, the convergence in Lebesgue spaces, the pointwise convergence, the convergence of variations, as well as the piecewise uniform convergence of regularized approximations, are obtained. In the case of functions of many variables, stabilizers containing the generalized variation [24] are often used. Here, the convergence in Lebesgue spaces, the pointwise convergence, and the convergence of generalized variations of regularized approximations are obtained [14-16,20-27]. Some results concerning the regularization with the use of variations for functions of many variables based on finite differences are presented in [20-23]. To obtain a uniform approximation of a solution that is continuous but nondifferentiable in the general case, stabilizers in the form of the norm of the Lipschitz space are applied $[15,16]$. The use of the norm of a Sobolev space with fractional derivatives as a stabilizer can be useful for reconstructing both continuous and discontinuous desired functions $[13,16]$. The results mentioned above are obtained for linear and nonlinear operator equations of the first kind. Similar investigations for inverse dynamic problems, in which it is required to qualitatively reconstruct unknown controls, are of a great interest.

In the present paper, it is shown that, using stabilizers in the form of the sum of the classical variation and the mean square norm of the admissible control in the problem of control reconstruction, one can obtain the pointwise convergence, the convergence in Lebesgue spaces, the convergence of classical variations, and the piecewise uniform convergence. The results obtained make possible the numerical reconstruction of the subtle structure of the desired control. In the paper, the subgradient projection method for obtaining minimizing sequences for the Tikhonov functional is described and substantiated. The two-stage finite-dimensional approximation of the problem is presented. At the first stage, the original infinite-dimensional problem is approximated by a simpler problem for some system of ordinary differential equations (the approximation is based on the method of separation of variables). The corresponding approximation theorem is proved. At the second stage, the problem for the system of ordinary differential equations is approximated by a finite-dimensional discrete problem. The carried out computational experiments show that the developed methods and algorithms can be applied to reconstruct the desired control and its subtle structure. The results of numerical simulation are presented. This paper continues investigations [28-33]. 


\section{PROBLEM STATEMENT}

We consider a controlled dynamical system, whose state at time $t$ from a given bounded time interval $T=\left[t_{0}, \vartheta\right]\left(t_{0}<\vartheta\right)$ is characterized by a function $y[t]=y(t, \cdot)$ defined in some domain $\Omega$ of Euclidean space $\mathbb{R}^{n}, n \geq 1$. The time evolution of the states $y[t]=y(t, \cdot)$ is described by the boundary value problem [34-36]

$$
\begin{gathered}
y_{t}=A y+f(t, x), \quad(t, x) \in Q=T \times \Omega, \\
y\left(t_{0}, x\right)=y_{0}(x), \quad x \in \Omega, \\
\sigma_{1} \frac{\partial y}{\partial N}+\sigma_{2} y=g(x) u(t), \quad t \in T, \quad x \in \Gamma=\partial \Omega,
\end{gathered}
$$

where $\sigma_{1}$ and $\sigma_{2}$ are nonnegative constants, $\sigma_{1}+\sigma_{2}>0 ; y_{0}$ is the initial state of the system; $f$ and $g$ are given functions; $\partial y / \partial N$ is the outer conormal derivative corresponding to the operator $A$; $u(t)=\left(u_{1}(t), \ldots, u_{m}(t)\right)$ is a vector control acting on the system at time $t \in T$; and $A$ is the linear self-adjoint differential operator

$$
A y=\sum_{i, j=1}^{n} \frac{\partial}{\partial x_{i}}\left(a_{i j}(x) \frac{\partial y}{\partial x_{j}}\right)-a(x) y .
$$

Admissible current values of the control action are subject to the constraint

$$
u(t) \in P \subset \mathbb{R}^{m}, \quad t \in T .
$$

Let the motion $y=y[t], t \in T$, of the controlled dynamical system be observed on the time interval $T$. Let the states $y[t]$ of the system be measured approximately at the corresponding current times $t \in T$, and let the results of these measurements $y_{\delta}[t]$ satisfy the condition

$$
\int_{T}\left\|y_{\delta}[t]-y[t]\right\|_{L_{2}(\Omega)}^{2} d t \leq \delta^{2},
$$

where $\delta$ is a numerical parameter characterizing the accuracy of measurements, $0 \leq \delta \leq \delta_{0}$.

The reconstruction problem consists in the approximate reconstruction (from the results $y_{\delta}=$ $y_{\delta}[t], t \in T$, of approximate measurements of the states of the system's observed motion $y=y[t]$, $t \in T$ ) of the control $u=u(t), t \in T$, generating the observed motion of the system. The result $u_{\delta}=u_{\delta}(t), t \in T$, of reconstructing the desired control $u=u(t), t \in T$, should be the more precise the smaller are the measurement errors:

$$
\int_{T}\left\|u_{\delta}(t)-u(t)\right\|_{R^{m}}^{2} d t \rightarrow 0, \quad \delta \rightarrow 0 .
$$

Further, the meaning of the approximate reconstruction $u_{\delta} \approx u$ will be varied and made more precise. Reconstruction algorithms providing, in addition to the traditional mean-square approximation (1.5), the approximation in some stronger sense resulting in the reconstruction of the subtle structure of the desired control will be suggested. Here, we will assume that the observer aiming to solve the reconstruction problem knows the a priori geometrical constraint on the set of admissible controls, the equations of the system's dynamics, and the initial state of the system. 
The problem under consideration can be informally interpreted as follows. In some body (domain) $\Omega$ with boundary $\Gamma$, the heat exchange process under the action of boundary heat forces with a time component $u$ is realized. This control component $u$ is a priori unknown and inaccessible for direct measurements. The process of heat distribution in the body $\Omega$ is observed during some finite time interval $T$. In the process, at current times $t \in T$, the temperature of the body $y[t]=y(t, x), x \in \Omega$, is measured with some error $\delta$. The result of these measurements is a function $y_{\delta}[t]=y_{\delta}(t, x), x \in \Omega$, satisfying estimate (1.4). It is required to reconstruct approximately the control realization $u$ generating the observed evolution of the temperature $y[t], t \in T$, from the received information $y_{\delta}[t], t \in T$, on the approximate temperature of the body on the time interval $T$. The reconstruction should be the more precise the smaller are the errors of measuring the temperature.

Let us pass to the mathematical formalization of the problem. Let $P$ be a convex compact set from $\mathbb{R}^{m}$. Let the set $U$ of all admissible controls in the problem be the set of all measurable and square integrable vector functions whose values for almost all $t \in T$ belong to the compact set $P$ :

$$
U=\{u \in E: u(t) \in P \text { for a.a. } t \in T\}, \quad E=L_{2}\left(T ; \mathbb{R}^{m}\right) .
$$

We assume that $\Omega$ is a bounded domain in $\mathbb{R}^{n}$ with piecewise smooth boundary $\Gamma$ [34-36], $f \in L_{2}(Q), g \in L_{2}^{m}(\Gamma), y_{0} \in L_{2}(\Omega)$, and the coefficients of the operator $A$ satisfy the following conditions in $\Omega: a_{i j}=a_{j i}, a \geq a_{0}=$ const $>0, a_{i j} \in L_{\infty}(\Omega), a \in L_{\infty}(\Omega)$, and, for $\sigma_{1}=0$, additionally $a_{i j} \in W_{p}^{1}(\Omega), p>n$.

It is known $[37,38]$ that, under the specified conditions on the parameters of boundary value problem (1.1)-(1.3), for any control $u \in E$, there exists a unique weak solution $y=y(t, x)=$ $y(t, x ; u),(t, x) \in Q$, to this boundary value problem from the space $C\left(T ; L_{2}(\Omega)\right)$. This solution is sometimes called the motion of dynamical system (1.1)-(1.3) generated by the control $u \in U$ and is denoted by the symbol $y=y[\cdot ; u]=y[t ; u], t \in T$.

Consider the set of all possible motions of system (1.1)-(1.3) corresponding to all possible controls

$$
Y=\{y=y[; u]: u \in U\} .
$$

For any motion $y \in Y$, we introduce the set of all admissible controls generating this motion

$$
U(y)=\{u \in U: y[\cdot ; u]=y\}
$$

and the set of all possible measurements of this motion

$$
Y_{\delta}(y)=\left\{y_{\delta} \in Z: \int_{T}\left\|y[t]-y_{\delta}[t]\right\|_{L_{2}(\Omega)}^{2} d t \leq \delta^{2}\right\}, \quad Z=L_{2}\left(T ; L_{2}(\Omega)\right) .
$$

We will find a solution to the reconstruction problem in the class of algorithms, each of which is identified with the family of mappings (methods)

$$
D=\left\{D_{\delta}: 0 \leq \delta \leq \delta_{0}\right\}, \quad D_{\delta}: Z \rightarrow E
$$

To formulate the problem, we introduce the notation:

$$
\begin{gathered}
r_{\delta}(y)=\sup \left\{\rho\left[D_{\delta}\left(y_{\delta}\right), U(y)\right]: y_{\delta} \in Y_{\delta}(y)\right\}, \\
\rho\left[D_{\delta}\left(y_{\delta}\right), U(y)\right]=\min \left\{\left\|D_{\delta}\left(y_{\delta}\right)-v\right\|_{E}: v \in U(y)\right\} .
\end{gathered}
$$


Problem. It is required to construct an algorithm $D$ that, for any observed motion of the system $y \in Y$, possesses the regularizing property

$$
r_{\delta}(y) \rightarrow 0, \quad \delta \rightarrow 0
$$

\section{PROPERTIES OF THE CONTROLLED SYSTEM}

Let us note a number of properties of the dynamical system and its motions. These properties will be used to substantiate the methods chosen for solving the reconstruction problem.

Lemma 2.1. The set $U$ is convex, bounded, closed, and weakly compact in $E$.

Lemma 2.2. If a sequence of controls $\left\{u_{k}\right\} \subset E$ converges weakly in $E$ to an element $u_{0} \in E$, then the corresponding sequence of motions $\left\{y\left[\cdot ; u_{k}\right]\right\}$ converges in $C\left(T ; L_{2}(\Omega)\right)$ to the motion $y\left[\cdot ; u_{0}\right]$.

Lemma 2.3. The mapping $B: E \ni u \rightarrow y[\cdot ; u] \in C\left(T ; L_{2}(\Omega)\right)$ is linear and compact.

Lemma 2.4. The set $Y$ is convex and compact in the space $C\left(T ; L_{2}(\Omega)\right)$.

Lemma 2.5. For every $y \in Y$, the set $U(y)$ is nonempty, convex, bounded, closed, and weakly compact in $E$; it contains a unique element $u^{*}(y)$ of minimal E-norm.

The proofs of the lemmas are similar to the proofs of analogous statements in $[31,32,38]$.

It follows from Lemma 2.3 that the mapping $B$ cannot have a continuous inverse mapping [39, p. 228]; therefore, the reconstruction problem under consideration is ill-posed and its solution requires the application of regularization methods.

\section{SOLUTION TO THE RECONSTRUCTION PROBLEM}

Consider one of the variants for solving the problem. Let us introduce the notation

$$
\begin{gathered}
F_{\alpha}^{*}(z)=\min \left\{F_{\alpha}(z, v): v \in U\right\}, \\
U_{\alpha}^{*}(z)=\left\{v \in U: F_{\alpha}(z, v)=F_{\alpha}^{*}(z)\right\}, \\
F_{\alpha}=F_{\alpha}(z, v)=\int_{T}\|y[t ; v]-z(t)\|_{L_{2}(\Omega)}^{2} d t+\alpha\|v\|_{E}^{2}, \\
\alpha=\text { const }>0 .
\end{gathered}
$$

Note some properties of functional (3.3), extremal problem (3.1), and the set of its solutions (the set of minimizing elements) (3.2).

Lemma 3.1. For any $\alpha>0$ and $z \in Z$, functional (3.3) is lower semicontinuous in $v$ with respect to weak convergence in $E$.

Lemma 3.2. For any $\alpha>0$ and $z \in Z$, extremal problem (3.1) is uniquely solvable; the set of its solutions $U_{\alpha}^{*}(z)$ consists of one element $u_{\alpha}^{*} \in U$.

Lemma 3.3. For any $\alpha>0$ and $z \in Z$, any minimizing sequence in problem (3.1) converges strongly in $E$ to the element $u_{\alpha}^{*}$.

The proofs of the lemmas are similar to the proofs of analogous statements in [31,32,38-42].

$$
\text { PROCEEDINGS OF The STEKLOV INSTITUTE OF MAThematics } \quad \text { Vol. } 280 \quad \text { Suppl. } 1 \quad 2013
$$


Now, we construct the algorithm solving the reconstruction problem. For any $\delta \in\left[0, \delta_{0}\right]$ and $z \in Z$, we define the realization (the value) of the method $D_{\delta}(z)$ by the rule

$$
D_{\delta}(z)=v \in U: F_{\alpha}^{*}(z) \leq F_{\alpha}(z, v) \leq F_{\alpha}^{*}(z)+\varepsilon,
$$

where $\varepsilon$ is a nonnegative parameter characterizing the accuracy by functional of the solution to extremal problem (3.1). The values $\alpha$ and $\varepsilon$ are parameters of the method (regularization parameters); they are chosen depending on the value $\delta$ of the accuracy of measurements.

Theorem 3.1. Let the regularization parameters $\alpha=\alpha(\delta)$ and $\varepsilon=\varepsilon(\delta)$ satisfy the following concordance conditions:

$$
\left(\varepsilon(\delta)+\delta^{2}\right) \alpha(\delta)^{-1} \rightarrow 0, \quad \varepsilon(\delta) \rightarrow 0, \quad \alpha(\delta) \rightarrow 0, \quad \delta \rightarrow 0 .
$$

Then, the algorithm D consisting of methods (3.4) solves the reconstruction problem on any observed motion $y \in Y$; i.e., $r_{\delta}(y) \rightarrow 0$ as $\delta \rightarrow 0$. In addition, for any realizations of measurements $y_{\delta} \in Y_{\delta}(y)$, the realizations $v_{\delta}=D_{\delta}\left(y_{\delta}\right)$ of methods (3.4) provide the strong convergence in $E$ $v_{\delta} \rightarrow u^{*}(y)$ as $\delta \rightarrow 0$.

The proof of the theorem is similar to the proof of analogous statement in [28-32].

Consider one more variant for solving the reconstruction problem.

Let $W$ be the Banach space of functions of bounded total variation [43-45]

$$
W=\{u \in E: V[u]<\infty\}, \quad\|u\|_{W}=\|u\|_{E}+V[u],
$$

where $V[u]$ is the total variation of the function $u: T \ni t \rightarrow u(t) \in \mathbb{R}^{m}$,

$$
V[u]=\sup \left\{\sum_{i=1}^{l}\left\|u\left(t_{i}\right)-u\left(t_{i-1}\right)\right\|_{\mathbb{R}^{m}}: \sigma \in \Sigma\right\} ;
$$

the supremum is taken over the set $\Sigma$ of all finite partitions $\sigma$ of the interval $T$,

$$
\sigma: t_{0}<t_{1}<\ldots<t_{l-1}<t_{l}=\vartheta .
$$

Note some properties of the space $W$ and its norm.

Lemma 3.4. The space $W$ is compactly embedded in $E$; i.e., the operator of embedding $W$ into $E$ is continuous and maps every bounded set from $W$ to a precompact set from $E$. Any closed bounded set from $W$ is compact in $E$.

Lemma 3.5. The pointwise limit of a bounded in $W$ sequence of functions is a function from $W$.

Lemma 3.6. The functional $V[\cdot]$ and the norm $\|\cdot\|_{W}$ are lower semicontinuous with respect to pointwise convergence of arguments; i.e., if a sequence of functions of bounded variation $\left\{v_{k}\right\}$ converges pointwise on $T$ to some function of bounded variation $v_{0}$, then $V\left[v_{0}\right] \leq \liminf V\left[v_{k}\right]$ and $\left\|v_{0}\right\|_{W} \leq \liminf \left\|v_{k}\right\|_{W}$.

Lemma 3.7. The functional $V[\cdot]$ and the norm $\|\cdot\|_{W}$ are lower semicontinuous with respect to the convergence in $E$ of bounded sequences from $W$; i.e., if $\left\{v_{k}\right\}$ is a bounded sequence from $W$ that converges in $E$ to some function $v_{0} \in E$, then $v_{0} \in W$ and $V\left[v_{0}\right] \leq \liminf V\left[v_{k}\right],\left\|v_{0}\right\|_{W} \leq$ $\liminf \left\|v_{k}\right\|_{W}$.

Lemma 3.8. For any closed ball $S_{r}\left[w_{0}\right]=\left\{w \in W:\left\|w-w_{0}\right\| \leq r\right\}$, the set $U \cap S_{r}\left[w_{0}\right]$ is convex, closed in $E$ and in $W$, compact, weakly compact in $E$, and compact with respect to pointwise convergence on $T$. 
The proofs of Lemmas 3.4-3.8 are similar to the proofs of analogous statements in [13,30-32]. Introduce the following notation:

$$
\begin{gathered}
\widehat{F}_{\alpha}^{*}(z)=\min \left\{\widehat{F}_{\alpha}(z, v): v \in U_{W}\right\}, \quad U_{W}=U \cap W, \\
\widehat{U}_{\alpha}^{*}(z)=\left\{v \in U_{W}: \widehat{F}_{\alpha}(z, v)=\widehat{F}_{\alpha}^{*}(z)\right\}, \\
\widehat{F}_{\alpha}=\widehat{F}_{\alpha}(z, v)=\int_{T}\|y[t ; v]-z(t)\|_{L_{2}(\Omega)}^{2} d t+\alpha \Lambda(v), \\
\Lambda(v)=\|v\|_{E}^{2}+V[v] .
\end{gathered}
$$

Note some properties of functional (3.8), extremal problem (3.6), and set (3.7) of its solutions.

Lemma 3.9. For any $\alpha>0$ and $z \in Z$, functional (3.8) is lower semicontinuous in $v$ with respect to the convergence in $E$ of bounded sequences from $W$.

Lemma 3.10. For any $\alpha>0$ and $z \in Z$, extremal problem (3.6) is uniquely solvable; the set of its solutions $\widehat{U}_{\alpha}^{*}(z)$ consists of one element $\widehat{u}_{\alpha}^{*} \in U_{W}$.

Lemma 3.11. For any $\alpha>0$ and $z \in Z$, any minimizing sequence in problem (3.6) converges strongly in $E$ to the element $\widehat{u}_{\alpha}^{*}$.

Let some functional $G$ be given on the set $U$. An element $\widehat{u}$ of a set $S \subseteq U$ satisfying the condition $G(\widehat{u})=\inf \{G(u): u \in S\}$ is called a $(G, S)$-normal element of the set $S$ and is denoted by the symbol $\widehat{u}(G, S)$.

Lemma 3.12. Let the set $S=U(y) \cap W$ be nonempty for $y \in Y$. Then, in the set $S$, there exists a unique $(\Lambda, S)$-normal element $\widehat{u}=\widehat{u}(\Lambda, S)$.

Now, we construct the algorithm solving the reconstruction problem. For any $\delta \in\left[0, \delta_{0}\right]$ and $z \in Z$, we define the realization (the value) of the method $D_{\delta}(z)$ by the rule

$$
D_{\delta}(z)=v \in U_{W}: \widehat{F}_{\alpha}^{*}(z) \leq \widehat{F}_{\alpha}(z, v) \leq \widehat{F}_{\alpha}^{*}(z)+\varepsilon
$$

where $\varepsilon$ is a nonnegative parameter characterizing the accuracy by functional of the solution to problem (3.6).

Define $Y_{*}=\{y \in Y: U(y) \cap W \neq \varnothing\}$.

Theorem 3.2. Let the regularization parameters $\alpha=\alpha(\delta)$ and $\varepsilon=\varepsilon(\delta)$ satisfy concordance conditions (3.5). Then, the algorithm $D$ consisting of methods (3.9) solves the reconstruction problem on any observed motion $y \in Y_{*}$; i.e., $r_{\delta}(y) \rightarrow 0$ as $\delta \rightarrow 0$. In addition, if $\widehat{u}$ is the $(\Lambda, S)$ normal element of the set $S=U(y) \cap W$, then, for any realizations of measurements $y_{\delta} \in Y_{\delta}(y)$, the realizations $v_{\delta}=D_{\delta}\left(y_{\delta}\right)$ of methods (3.9) satisfy the following convergences as $\delta \rightarrow 0:(1) v_{\delta} \rightarrow \widehat{u}$ strongly in $E$; (2) $v_{\delta} \rightarrow \widehat{u}$ in $\mathbb{R}^{m}$ pointwise on $T ;(3) V\left[v_{\delta}\right] \rightarrow V[\widehat{u}] ;(4) v_{\delta}(t) \rightarrow \widehat{u}(t)$ in $\mathbb{R}^{m}$ uniformly in $t$ on any interval not containing the discontinuity points of the function $\widehat{u}$.

The proofs of Lemmas 3.9-3.12 and Theorem 3.2 are similar to the proofs of analogous statements in [28-32].

\section{RECONSTRUCTION OF CONTROLS DISTRIBUTED ALONG THE BOUNDARY}

Consider the case of controls distributed along the boundary: $u=u(t, x), t \in T, x \in \Gamma_{*} \subseteq \Gamma$. Let, for simplicity, $n=2, m=1$. Assume that some part $\Gamma_{*}$ of the boundary $\Gamma$ is parameterized by a variable $s \in\left[s_{1}, s_{2}\right]$ and that admissible controls are concentrated on this parameterized part. 
Let $\Pi=T \times\left[s_{1}, s_{2}\right], E=L_{2}(\Pi)$, and $U=\{u \in E: u(t, s) \in P$ for a.a. $(t, s) \in \Pi\}$. As a stabilizer, we consider the functional $\Lambda(v)=\|v\|_{E}^{2}+\|v\|_{W}$, where $\|\cdot\|_{W}$ is the norm in the Banach space $W$ of functions $v=v(t, s),(t, s) \in \Pi$, with finite total variation $V H(v, \Pi)$ [23, p. 89]. Repeating the above scheme for solving the reconstruction problem and using results [23, pp. 89-91], we obtain the following statement, which is similar to Theorem 3.2.

Theorem 4.1. Let the regularization parameters $\alpha=\alpha(\delta)$ and $\varepsilon=\varepsilon(\delta)$ satisfy concordance conditions (3.5). Let $y \in Y_{*}$, and let the control $\widehat{u}$ be the $(\Lambda, S)$-normal element of the set $S=$ $U(y) \cap W$. Let this control be "essentially unique" [23, p. 90] and continuous in a domain $\Pi_{*} \subseteq \Pi$ together with the function $\pi(t, x)=V H\left(\widehat{u}, \Pi_{t_{0} s_{1}}^{t, s}\right)$ [23, p. 89]. Then, the algorithm $D$ consisting of methods (3.9) solves the reconstruction problem on any observed motion $y \in Y_{*}$; i.e., $r_{\delta}(y) \rightarrow 0$ as $\delta \rightarrow 0$. In addition, for any realizations of measurements $y_{\delta} \in Y_{\delta}(y)$, the realizations $v_{\delta}=D_{\delta}\left(y_{\delta}\right)$ of methods (3.9) satisfy the following convergences as $\delta \rightarrow 0:(1) v_{\delta} \rightarrow \widehat{u}$ strongly in $E ;(2) v_{\delta} \rightarrow \widehat{u}$ in $\mathbb{R}$ pointwise on $\Pi ; \quad(3) V H\left(v_{\delta}, \Pi\right) \rightarrow V H(\widehat{u}, \Pi) ; \quad(4) v_{\delta}(t, s) \rightarrow \widehat{u}(t, s)$ in $\mathbb{R}$ uniformly in $(t, s)$ from any closed subdomain $\Pi^{*} \subset \Pi_{*}$.

A similar statement is valid when the total variation $V H(v, \Pi)$ is replaced by the Arzela variation $V A(v, \Pi)$ [23, pp. 89-91]. Then, the concluding part of the statement is reformulated as follows: (1) $v_{\delta} \rightarrow \widehat{u}$ strongly in $E ;(2) v_{\delta} \rightarrow \widehat{u}$ in $\mathbb{R}$ at continuity points of the function $\widehat{u} ;(3) V A\left(v_{\delta}, \Pi\right) \rightarrow$ $V A(\widehat{u}, \Pi) ;(4) v_{\delta}(t, s) \rightarrow \widehat{u}(t, s)$ in $\mathbb{R}$ uniformly in $(t, s)$ from any closed subdomain $\Pi^{*} \subset \Pi_{*}$.

\section{CONSTRUCTION OF MINIMIZING SEQUENCES}

To solve approximately problem (3.6), i.e., to obtain $\varepsilon$-optimal solutions satisfying condition (3.9), it is necessary to construct minimizing sequences for problem (3.6). For constructing such sequences, one can use gradient and subgradient methods [39-42]. Let us substantiate some auxiliary statements in this direction. The investigation of similar questions for problem (3.1) is simpler, since there is no variation in the target functional.

First, we note some differential properties of the functional $\widehat{F}_{\alpha}$. We represent it as the sum of the corresponding terms

$$
\begin{gathered}
\widehat{F}_{\alpha}(z, u)=J_{1}(z, u)+\alpha J_{2}(u)+\alpha J_{3}(u) \\
J_{1}(z, u)=\int_{T}\|y[t ; u]-z(t)\|_{L_{2}(\Omega)}^{2} d t, \quad J_{2}(u)=\|u\|_{E}^{2}, \quad J_{3}(u)=V[u] .
\end{gathered}
$$

The functionals $J_{1}$ and $J_{2}$ are Fréchet differentiable with respect to the variable $u$ at any point $u \in E$ (as functionals $E \rightarrow \mathbb{R}$ ) and at any point $u \in W$ (as functionals $W \rightarrow \mathbb{R}$ ), the functional $J_{3}$ is neither Fréchet nor Gateaux differentiable on $W$ (for example, it is not Gateaux differentiable at the point $u=0$ ). The functional $\widehat{F}_{\alpha}$ is convex and continuous on $W$; therefore, it is subdifferentiable with respect to the variable $u$ on $W[40]$

$$
\partial_{u} \widehat{F}_{\alpha}(z, u)=J_{1 u}^{\prime}(z, u)+\alpha J_{2}^{\prime}(u)+\alpha \partial J_{3}(u) .
$$

The practical application of subgradient methods to the minimization of the functional $\widehat{F}_{\alpha}$ meets difficulties concerning the complexity of calculating the subdifferential $\partial J_{3}(u)$ and describing the conjugate space $W^{*}$. Some possible approaches to the numerical realization of the minimization problem can be connected with the approximation of the functional $J_{3}$ by appropriate differentiable functionals [13,23-27]. In the present paper, the approach from [14-19] is realized. This approach is based on replacing the space $W$ by some Hilbert space, for example, by the Sobolev space 
$H=W_{2}^{1}(T)^{m}$ that should be embedded into $W$ and should approximate its elements sufficiently well. This allows us to use the methods applicable for Hilbert spaces and to simplify calculating gradients and subgradients of the target functional.

Consider the auxiliary extremal problem

$$
F_{\alpha}^{\circ}(z)=\inf \left\{\widehat{F}_{\alpha}(z, v): v \in U_{H}\right\}, \quad U_{H}=U \cap H .
$$

Let us formulate some statements related to this problem.

Lemma 5.1. The inclusion $H \subset W$ is valid and

$$
V[u]=\int_{T}\|\dot{u}(t)\|_{\mathbb{R}^{m}} d t
$$

for every element $u \in H$.

Lemma 5.2. For every element $u \in U_{W}$, there exists a sequence of elements $\left\{u_{k}\right\} \subset U_{H}$ such that $u_{k} \rightarrow u$ strongly in $E$ and

$$
\limsup V\left[u_{k}\right] \leq V[u]
$$

Lemma 5.3. For any $\alpha>0$ and $z \in Z, F_{\alpha}^{\circ}(z)=\widehat{F}_{\alpha}^{*}(z)$.

Lemma 5.4. For any $\alpha>0$ and $z \in Z$, any minimizing sequence of problem (5.1) converges strongly in $E$ to the element $\widehat{u}_{\alpha}^{*}$.

Lemmas 5.1 and 5.2 are proved in [30]. The proofs of Lemmas 5.3 and 5.4 are similar to the proofs of analogous statements in [30].

Now, we investigate the question about the differentiability of the functional $\widehat{F}_{\alpha}: H \rightarrow \mathbb{R}$.

For the increment of the functional $J_{1}$ in the variable $u$, the following representation (it is derived similarly to $[39$, Ch. 8 , Sect. 7$]$ ) is true:

$$
\begin{gathered}
J_{1}(z, u+h)-J_{1}(z, u)=\langle b(\psi), h\rangle_{E}+o\left(\|h\|_{E}\right), \\
\left|o\left(\|h\|_{E}\right)\right| \leq C\|h\|_{E}^{2} \leq C\|h\|_{H}^{2}, \\
b(\psi)=\sigma_{2}^{-1}\left\langle g, \frac{\partial \psi}{\partial N}\right\rangle_{L_{2}(\Gamma)}=\sigma_{2}^{-1}\left(\left\langle g_{1}, \frac{\partial \psi}{\partial N}\right\rangle_{L_{2}(\Gamma)}, \ldots,\left\langle g_{m}, \frac{\partial \psi}{\partial N}\right\rangle_{L_{2}(\Gamma)}\right) \text { for } \sigma_{1}=0, \\
b(\psi)=-\sigma_{1}^{-1}\langle g, \psi\rangle_{L_{2}(\Gamma)}=-\sigma_{1}^{-1}\left(\left\langle g_{1}, \psi\right\rangle_{L_{2}(\Gamma)}, \ldots,\left\langle g_{m}, \psi\right\rangle_{L_{2}(\Gamma)}\right) \text { for } \sigma_{1} \neq 0,
\end{gathered}
$$

$C$ is some positive constant not depending on $u \in E$ and $h \in E, \psi=\psi(\cdot ; z, u)$ is a solution to the conjugate problem

$$
\begin{gathered}
\psi_{t}=-A \psi+2(y(t, x ; u)-z(t, x)), \quad(t, x) \in Q, \\
\psi(\vartheta, x)=0, \quad x \in \Omega, \\
\sigma_{1} \frac{\partial \psi}{\partial N}+\sigma_{2} \psi=0, \quad t \in T, \quad x \in \Gamma .
\end{gathered}
$$

It is clear that the functional $\eta_{u}$ acting by the rule $\eta_{u}(h)=\langle b(\psi), h\rangle_{E}$ is a linear continuous functional on $E, W$, and $H$. It follows from (5.2) that this functional is the Fréchet derivative of the functional $J_{1}(z, \cdot): H \rightarrow \mathbb{R}$ at the point $u \in H$; i.e.,

$$
J_{1 u}^{\prime}(z, u)=\eta_{u} \in H^{*}, \quad \eta_{u}(h)=\langle b(\psi), h\rangle_{E} \quad \forall h \in H .
$$


By the Riesz theorem about the representation of a linear continuous functional on a Hilbert space, for the functional $\eta_{u}$, there exists a unique element $a_{u}^{(1)} \in H$ providing the relation

$$
\eta_{u}(h)=\langle b(\psi), h\rangle_{E}=\left\langle a_{u}^{(1)}, h\right\rangle_{H} \quad \forall h \in H .
$$

It follows from (5.6) and (5.7) that the element $a_{u}^{(1)} \in H$ is the gradient of the functional $J_{1}(z, \cdot): H \rightarrow \mathbb{R}$ at the point $u \in H$; i.e.,

$$
\nabla_{u} J_{1}(z, u)=a_{u}^{(1)}, \quad \eta_{u}(h)=\langle b(\psi), h\rangle_{E}=\left\langle a_{u}^{(1)}, h\right\rangle_{H} \quad \forall h \in H .
$$

Thus, to calculate the derivative $J_{1 u}^{\prime}(z, u)$, one should successively solve two problems: first, find the solution $y=y(\cdot ; u)$ to boundary value problem $(1.1)-(1.3)$; second, find the solution $\psi=$ $\psi(\cdot ; z, u)$ to boundary value problem (5.3)-(5.5) and, by formula (5.6), the desired functional $\eta_{u}$. To find the gradient $\nabla_{u} J_{1}(z, u)$, one should find an element $a_{u}^{(1)} \in H$ providing representation (5.8).

Let us formulate this result as a lemma.

Lemma 5.5. For every element $z \in Z$, the functional $J_{1}(z, \cdot): H \rightarrow \mathbb{R}$ is continuous and Fréchet differentiable at any point $u \in H$. The Fréchet derivative $J_{1 u}^{\prime}(z, u) \in H^{*}$ at the point $u \in H$ is found from equality (5.6). The gradient $\nabla_{u} J_{1}(z, u) \in H$ at the point $u \in H$ is found from (5.8). In addition, under the specified conditions on the initial data of the problem, the derivative $J_{1 u}^{\prime}(z, \cdot)$ and gradient $\nabla_{u} J_{1}(z, \cdot)$ satisfy the Lipschitz condition on $U_{H}$; i.e., there exists a number $L \geq 0$ such that, for any $u \in U_{H}$ and $v \in U_{H}$, the following inequalities hold:

$$
\begin{gathered}
\left\|J_{1 u}^{\prime}(z, u)-J_{1 u}^{\prime}(z, v)\right\|_{H^{*}} \leq L\|u-v\|_{H}, \\
\left\|\nabla_{u} J_{1}(z, u)-\nabla_{u} J_{1}(z, v)\right\|_{H} \leq L\|u-v\|_{H} .
\end{gathered}
$$

The differentiability of the functional $J_{1}$ with respect to the variable $u$ implies its subdifferentiability; the subdifferential $\partial_{u} J_{1}(z, u)$ consists of one element $\eta_{u}, \partial_{u} J_{1}(z, u)=\left\{\eta_{u}\right\}[40$, pp. 58, 208, 227].

Lemma 5.6. The functional $J_{2}: H \rightarrow R$ is continuous and Fréchet differentiable at any point $u \in H$. The Fréchet derivative $J_{2}^{\prime}(u) \in H^{*}$ at the point $u \in H$ is defined by the equality

$$
J_{2}^{\prime}(u)=\varphi_{u} \in H^{*}, \quad \varphi_{u}(h)=\langle 2 u, h\rangle_{E} \quad \forall h \in H .
$$

The gradient $\nabla J_{2}(u) \in H$ at the point $u \in H$ is defined by the equality

$$
\nabla J_{2}(u)=a_{u}^{(2)}, \quad \varphi_{u}(h)=\langle 2 u, h\rangle_{E}=\left\langle a_{u}^{(2)}, h\right\rangle_{H} \quad \forall h \in H .
$$

The proof of a similar statement is presented, for example, in [39, Ch. 8, Sect. 3]. The differentiability of the functional $J_{2}$ implies its subdifferentiability; the subdifferential $\partial J_{2}(u)$ consists of one element $\varphi_{u}, \partial J_{2}(u)=\left\{\varphi_{u}\right\} \quad[40$, pp. 58, 208, 227].

In general, the functional $J_{3}: H \rightarrow \mathbb{R}$ is neither Fréchet nor Gateaux differentiable on $H$. For example, it does not even have the weak Gateaux derivative at the point $u=0$. However, it is continuous and convex on $H$ and, therefore, subdifferentiable at every point $u \in H[40$, Ch. 4, Sect. 4.2]; moreover, for the subdifferential, we have the equality

$$
\partial J_{3}(u)=\left\{\varphi \in H^{*}: \varphi(v-u) \leq V[v]-V[u] \forall v \in H\right\} .
$$

Note one useful statement (see, for example, [40, Ch. 4, Sect. 4.2, Sect. 4.4]): the functional $J_{3}: H \rightarrow \mathbb{R}$ is Gateaux differentiable at the point $u \in H$ if and only if its subdifferential $\partial J_{3}(u)$ at 
this point contains exactly one element $\varphi_{u} \in H^{*}$, which is the Gateaux derivative at this point; i.e., $J_{3}^{\prime}(u)=\varphi_{u}$.

Lemma 5.7. The functional $J_{3}: H \rightarrow \mathbb{R}$ is Gateaux differentiable at the point $u \in H$ if and only if the Lebesgue measure of the set $T_{u}=\{t \in T: \dot{u}(t)=0\}$ is zero. In the case of differentiability, the Gateaux derivative $J_{3}^{\prime}(u)$ is found from the equality

$$
J_{3}^{\prime}(u)(h)=\left\langle\frac{\dot{u}}{\|\dot{u}\|_{\mathbb{R}^{m}}}, \dot{h}\right\rangle_{E} \quad \forall h \in H .
$$

Lemma 5.8. If the functional $J_{3}: H \rightarrow \mathbb{R}$ is Gateaux differentiable at the point $u \in H$, then it is Fréchet differentiable at the same point; in addition, the Gateaux and Fréchet derivatives at this point coincide as elements of $H^{*}$.

At the points $u \in H$ where the functional $J_{3}: H \rightarrow \mathbb{R}$ is not Gateaux differentiable, the subdifferential $\partial J_{3}(u)$ consists of more than one element. Indeed, the subdifferential is not empty and, if it contained only one element, then the functional $J_{3}$ would be Gateaux differentiable at the point $u$ [40, p. 208]. In fact, at such points, the subdifferential $\partial J_{3}(u)$ contains an infinite number of different elements, since, if the set $\partial J_{3}(u)$ contains at least two different elements $w_{1}$ and $w_{2}$, then, by virtue of its convexity, it must contain all elements of the form $w_{\lambda}=\lambda \times w_{1}+(1-\lambda) \times w_{2}$, $0 \leq \lambda \leq 1$. It follows from the general theorems that the set $\partial J_{3}(u)$ is convex, bounded, and *-weakly compact in $H$ [39, p. 199; 40, p. 210]. By the Riesz theorem about the representation of a linear continuous functional on a Hilbert space, the set $\partial J_{3}(u)$ from $H^{*}$ is related to the convex, bounded, and weakly compact set of subgradients $I\left(\partial J_{3}(u)\right)$ from $H$, where $I$ is the linear isometric isomorphism between $H^{*}$ and $H$ defined by the Riesz theorem.

The relation representing the subdifferential $\partial J_{3}(u)$, in view of Lemma 5.1, takes the form

$$
\partial J_{3}(u)=\left\{\varphi \in H^{*}: \varphi(v-u) \leq \int_{T}\|\dot{v}(\tau)\|_{\mathbb{R}^{m}} d \tau-\int_{T}\|\dot{u}(\tau)\|_{\mathbb{R}^{m}} d \tau \quad \forall v \in H\right\} .
$$

The set of subgradients $D J_{3}(u)$ of the subdifferential $\partial J_{3}(u)$ is defined by the equality

$$
\begin{gathered}
D_{3}(u)=I\left(\partial J_{3}(u)\right) \\
D J_{3}(u)=\left\{w \in H:\langle w, v-u\rangle_{H} \leq \int_{T}\|\dot{v}(\tau)\|_{\mathbb{R}^{m}} d \tau-\int_{T}\|\dot{u}(\tau)\|_{\mathbb{R}^{m}} d \tau \quad \forall v \in H\right\} .
\end{gathered}
$$

If we consider only functionals $\varphi_{g} \in H^{*}$ of the form

$$
\varphi_{g}(v)=\langle\dot{g}, \dot{v}\rangle_{E}=\int_{T}\langle\dot{g}(\tau), \dot{v}(\tau)\rangle_{\mathbb{R}^{m}} d \tau, \quad v \in H
$$

then it is easy to verify that, at the point $u \in H$ with meas $T_{u}>0$, the subdifferential $\partial J_{3}(u)$ contains all functionals $\varphi_{g} \in H^{*}$ with generating elements $g \in H$ of the form

$$
\dot{g}(t)=\frac{\dot{u}(t)}{\|\dot{u}(t)\|_{\mathbb{R}^{m}}} \text { for } t \in T \backslash T_{u}, \quad\|\dot{g}(t)\|_{\mathbb{R}^{m}} \leq 1 \text { for } t \in T_{u} .
$$


It turns out that the subdifferential $\partial J_{3}(u)$ consists exactly of all such functionals $\varphi_{g}$ of form (5.9) with generating elements $g$ of form (5.10).

Lemma 5.9. The subdifferential $\partial J_{3}(u)$ consists exactly of all functionals $\xi_{w} \in H^{*}$ of the form

$$
\xi_{w}(h)=\langle\dot{w}, \dot{h}\rangle_{E}=\int_{T}\langle\dot{w}(t), \dot{h}(t)\rangle_{\mathbb{R}^{m}} d t \quad \forall h \in H
$$

with generating elements $w$ from the set

$$
\Xi_{u}=\left\{w \in H: \dot{w}(t)=\frac{\dot{u}(t)}{\|\dot{u}(t)\|_{\mathbb{R}^{m}}} \text { for } t \in T \backslash T_{u}, \quad\|\dot{w}(t)\|_{\mathbb{R}^{m}} \leq 1 \text { for } t \in T_{u}\right\}
$$

i.e.,

$$
\partial J_{3}(u)=\left\{\xi_{w}: w \in \Xi_{u}\right\} .
$$

At every point $u \in H$, the set $D J_{3}(u)$ has the following structure:

$$
D J_{3}(u)=\left\{w \in H: \int_{T} w(t) d t=0, \dot{w}(t)=\int_{t_{0}}^{t} w(\tau) d \tau+\dot{g}(t), \quad g \in \Xi_{u}\right\} .
$$

Lemmas 5.6-5.9 are proved in [30].

By the Moreau-Rockafellar theorem [40], we have

$$
\begin{gathered}
\partial_{u} F_{\alpha}(z, u)=J_{1 u}^{\prime}(z, u)+\alpha J_{2}^{\prime}(u)+\alpha \partial J_{3}(u), u \in H, \\
D_{u} F_{\alpha}(z, u)=\nabla_{u} J_{1}(z, u)+\alpha \nabla J_{2}(u)+\alpha D J_{3}(u), u \in H .
\end{gathered}
$$

To construct minimizing sequences in problem (3.6), we apply the subgradient projection method [39, Ch. 5, Sect. $3 ; 41]$

$$
u^{(k+1)}=\operatorname{Pr}\left(u^{(k)}-\beta_{k} v_{k}\right), \quad \beta_{k}>0, \quad v_{k} \in D_{u} F_{\alpha}\left(z, u^{(k)}\right), \quad k=0,1,2, \ldots,
$$

where $u^{(0)}$ is an initial approximation from $U_{H}, \operatorname{Pr}(w)$ is the projection of the point $w \in H$ onto the set $U_{H}$ (since the set $U_{H}$ is convex and closed in $H$, the projection exists and is unique [39, Ch. 8, Sect. 4]), and the parameters of the method $\beta_{k}$ should be chosen in an appropriate way.

Theorem 5.1. Let $u^{(0)}$ be an arbitrary initial approximation from $U_{H}$ in iterative process $(5.11)$, let $v_{k}$ be an arbitrary subgradient from $D_{u} F_{\alpha}\left(z, u^{(k)}\right)$, and let the parameters of the method $\beta_{k}$ satisfy the condition

$$
\begin{gathered}
\beta_{k}=1 \quad \text { if } \quad v_{k}=0 ; \quad \beta_{k}=\gamma_{k} /\left\|v_{k}\right\|_{H} \quad \text { if } \quad v_{k} \neq 0 \\
\gamma_{k}>0, \quad \gamma_{k} \rightarrow 0, \quad \sum_{k=0}^{\infty} \gamma_{k}=\infty .
\end{gathered}
$$

Then, (1) $\widehat{F}_{\alpha}\left(z, u_{*}^{(k)}\right) \rightarrow F_{\alpha}^{\circ}(z)=\widehat{F}_{\alpha}^{*}(z)$, where $\widehat{F}_{\alpha}\left(z, u_{*}^{(k)}\right)=\min \left\{\widehat{F}_{\alpha}\left(z, u^{(i)}\right): i \in \overline{0, k}\right\} ;(2)$ the minimizing sequence $\left\{u_{*}^{(k)}\right\} \subset U_{H}$ of problem (5.1) converges strongly in $E$ to the element $u_{\alpha}^{*}$; (3) $V\left[u_{*}^{(k)}\right] \rightarrow V\left[u_{\alpha}^{*}\right]$.

The proof of the theorem is similar to the proof of Theorem 2 from [32]. 


\section{APPROXIMATION OF THE PROBLEM}

To approximate the problem, we apply the method of separation of variables. The solution to boundary value problem (1.1)-(1.3) is presented in the form of the Fourier series

$$
\begin{gathered}
y=y(t, x ; u)=\sum_{i=1}^{\infty} y_{i}(t) \omega_{i}(x), \quad t \in T, \quad x \in \Omega, \\
\dot{y}_{i}(t)=-\lambda_{i} y_{i}(t)+f_{i}(t)+g^{(i)} u(t), \quad t \in T, \quad y_{i}\left(t_{0}\right)=y_{0 i}, \quad i=1,2,3, \ldots, \\
y_{i}(t)=y_{i}(t ; u)=y_{0 i} \exp \left(-\lambda_{i}\left(t-t_{0}\right)\right)+\int_{t_{0}}^{t} f_{i}(\tau) \exp \left(-\lambda_{i}(t-\tau)\right) d \tau+g^{(i)} \int_{t_{0}}^{t} u(\tau) \exp \left(-\lambda_{i}(t-\tau)\right) d \tau,
\end{gathered}
$$

where $y_{0 i}=\left\langle y_{0}, \omega_{i}\right\rangle_{L_{2}(\Omega)}, f_{i}(t)=\left\langle f(t, \cdot), \omega_{i}\right\rangle_{L_{2}(\Omega)}, g^{(i)}=\left(\left\langle g_{1}, \Pi\left(\omega_{i}\right)\right\rangle_{L_{2}(\Gamma)}, \ldots,\left\langle g_{m}, \Pi\left(\omega_{i}\right)\right\rangle_{L_{2}(\Gamma)}\right)$, $\Pi\left(\omega_{i}\right)=-\sigma_{2}^{-1} \partial \omega_{i} / \partial N$ for $\sigma_{1}=0, \Pi\left(\omega_{i}\right)=\sigma_{1}^{-1} \omega_{i}$ for $\sigma_{1} \neq 0$, and $\left\{\lambda_{i}, \omega_{i}: i \in \mathbb{N}\right\}$ is a solution in $W_{2}^{1}(\Omega)$ of the spectral problem

$$
A \omega=-\lambda \omega \text { in } \Omega, \quad \sigma_{1} \frac{\partial \omega}{\partial N}+\sigma_{2} \omega=0 \text { on } \Gamma, \quad\langle\omega, \omega\rangle_{L_{2}(\Omega)}=1 .
$$

It is known [34-36] that the spectral problem is solvable for a countable set of real positive numbers $\lambda=\lambda_{i}, i \in \mathbb{N}$, each of which has finite multiplicity. These numbers can be ordered (taking into account their multiplicities) in the increasing order

$$
0<\lambda_{1} \leq \lambda_{2} \leq \ldots \leq \lambda_{i} \leq \ldots, \quad \lambda_{i} \rightarrow \infty \text { as } i \rightarrow \infty
$$

The eigenfunctions $\omega_{i}$ corresponding to the eigenvalues $\lambda_{i}$ form an orthonormal basis in $L_{2}(\Omega)$, a basis in $\stackrel{\circ}{W}_{2}^{1}(\Omega)$ and $W_{2,0}^{2}(\Omega)$ if $\sigma_{1}=0$, and a basis in $W_{2}^{1}(\Omega)$ if $\sigma_{1} \neq 0$.

For any $t \in T$ and $u \in U$, the following estimate with convergent series [38] is valid:

$$
\begin{gathered}
\|y(t, \cdot ; u)\|_{L_{2}(\Omega)}^{2}=\sum_{i=1}^{\infty} y_{i}(t ; u)^{2} \leq 3 \sum_{i=1}^{\infty} y_{0 i}^{2}+\frac{3}{2} \sum_{i=1}^{\infty}\left(\lambda_{i}\right)^{-1} \int_{T} f_{i}(\tau)^{2} d \tau \\
+3 m C_{P}^{2}\left(\sum_{i=1}^{m} \sum_{j=1}^{\infty}\left\langle g_{i}, \Pi\left(\omega_{j}\right)\right\rangle_{L_{2}(\Gamma)}^{2}\left(\lambda_{j}\right)^{-2}\right)<\infty \\
C_{P}=\sup \left\{\|w\|_{\mathbb{R}^{m}}: w \in P\right\} .
\end{gathered}
$$

This estimate implies, in particular, the convergence of the residual series $R_{p} \rightarrow 0$ as $p \rightarrow \infty$,

$$
R_{p}=3 \sum_{i=p}^{\infty} y_{0 i}^{2}+\frac{3}{2} \sum_{i=p}^{\infty}\left(\lambda_{i}\right)^{-1} \int_{T} f_{i}(\tau)^{2} d \tau+3 m C_{P}^{2}\left(\sum_{i=1}^{m} \sum_{j=p}^{\infty}\left\langle g_{i}, \Pi\left(\omega_{j}\right)\right\rangle_{L_{2}(\Gamma)}^{2}\left(\lambda_{j}\right)^{-2}\right) .
$$

Let us fix some $p \in \mathbb{N}$ and consider the extremal problem

$$
\begin{gathered}
\tilde{F}_{\alpha}^{(p)}(z)=\min \left\{F_{\alpha}^{(p)}(z, v): v \in U_{W}\right\}, \\
F_{\alpha}^{(p)}=F_{\alpha}^{(p)}(z, v)=J_{1}^{(p)}(z, v)+\alpha \Lambda(v),
\end{gathered}
$$




$$
J_{1}^{(p)}(z, v)=\sum_{i=1}^{p} \int_{T}\left(y_{i}(t ; v)-z_{i}(t)\right)^{2} d t, \quad z_{i}(t)=\left\langle z(t, \cdot), \omega_{i}\right\rangle_{L_{2}(\Omega)}, \quad z \in Z .
$$

For any $\alpha>0$ and $z \in Z$, extremal problem (6.1) has a unique solution $u_{\alpha}^{(p)}(z) \in U_{W}$, and any minimizing sequence in problem (6.1) converges strongly in $E$ to the element $u_{\alpha}^{(p)}(z)$.

Consider the following algorithm for solving the original reconstruction problem:

$$
D=\left\{D_{\delta}^{(p)}: 0 \leq \delta \leq \delta_{0}, p \in \mathbb{N}\right\}, \quad D_{\delta}^{(p)}: Z \rightarrow E .
$$

For any $\delta \in\left[0, \delta_{0}\right], p \in \mathbb{N}$, and $z \in Z$, we define the realization of the method

$$
D_{\delta}^{(p)}(z)=v \in U_{W}: \widetilde{F}_{\alpha}^{(p)}(z) \leq F_{\alpha}^{(p)}(z, v) \leq \widetilde{F}_{\alpha}^{(p)}(z)+\varepsilon,
$$

where $\varepsilon$ is a nonnegative parameter characterizing the accuracy by functional of the solution to extremal problem (6.1).

Theorem 6.1. Let the regularization parameters $\alpha=\alpha(\delta), p=p(\delta)$, and $\varepsilon=\varepsilon(\delta)$ satisfy the concordance conditions

$$
\left(\varepsilon(\delta)+\delta^{2}+R_{p(\delta)}\right) \quad \alpha(\delta)^{-1} \rightarrow 0, \varepsilon(\delta) \rightarrow 0, \quad \alpha(\delta) \rightarrow 0, \quad p(\delta) \rightarrow \infty, \quad \delta \rightarrow 0 .
$$

Then, the algorithm D consisting of methods (6.2) solves the reconstruction problem on any observed motion $y \in Y_{*}$; i.e., $r_{\delta}(y) \rightarrow 0$ as $\delta \rightarrow 0$. In addition, if $\widehat{u}$ is the $(\Lambda, S)$-normal element of the set $S=U(y) \cap W$, then, for any realizations of measurements $y_{\delta} \in Y_{\delta}(y)$, the realizations $v_{\delta}=D_{\delta}^{(p(\delta))}\left(y_{\delta}\right)$ of methods (6.2) satisfy the following convergences as $\delta \rightarrow 0:(1) V\left[v_{\delta}\right] \rightarrow V[\widehat{u}]$; (2) $v_{\delta} \rightarrow \widehat{u}$ strongly in $E$; (3) $v_{\delta} \rightarrow \widehat{u}$ in $\mathbb{R}^{m}$ pointwise on $T ;(4) v_{\delta}(t) \rightarrow \widehat{u}(t)$ in $\mathbb{R}^{m}$ uniformly in $t$ from any interval not containing the discontinuity points of the function $\widehat{u}$.

The proof of the theorem is similar to the proof of Theorem 3.2.

Now, we specify the method for constructing minimizing sequences in problem (6.1). This method is similar to the one presented in Section 5 .

Consider the new auxiliary extremal problem

$$
\stackrel{\circ}{F}_{\alpha}^{(p)}(z)=\inf \left\{F_{\alpha}^{(p)}(z, u): u \in U_{H}\right\} .
$$

As in Section 5 , one can show that $\stackrel{\circ}{F}_{\alpha}^{(p)}(z)=\widetilde{F}_{\alpha}^{(p)}(z)$ and any minimizing sequence in problem (6.3) converges strongly in $E$ to the element $u_{\alpha}^{(p)}(z)$.

The functional $F_{\alpha}^{(p)}: H \rightarrow \mathbb{R}$ is neither Fréchet nor Gateaux differentiable with respect to the variable $u$ on $H$, but it is subdifferentiable at every point $u \in H$

$$
\partial_{u} F_{\alpha}^{(p)}(z, u)=J_{1 u}^{(p) \prime}(z, u)+\alpha J_{2}^{\prime}(u)+\alpha \partial J_{3}(u) .
$$

The Fréchet derivative $J_{2}^{\prime}(u)$ and subdifferential $\partial J_{3}(u)$ are found above. Let us formulate the corresponding statement about the derivative of the functional $J_{1}^{(p)}(z, u)$.

Lemma 6.1. For every element $z \in Z$, the functional $J_{1}(z, \cdot): H \rightarrow \mathbb{R}$ is continuous and Fréchet differentiable at any point $u \in H$. The Fréchet derivative $J_{1 u}^{(p) \prime}(z, u)$ at the point $u \in H$ is defined by the equality

$$
J_{1 u}^{(p) \prime}(z, u)=\eta_{u} \in H^{*}, \quad \eta_{u}(h)=\left\langle b_{p}(\psi), h\right\rangle_{E} \quad \forall h \in H,
$$


where $b_{p}(\psi)=\psi_{1} g^{(1)}+\ldots+\psi_{p} g^{(p)}, \psi^{(p)}=\left(\psi_{1}, \ldots, \psi_{p}\right)$, is the solution to the conjugate problem

$$
\dot{\psi}_{i}(t)=\lambda_{i} \psi_{i}(t)-2\left(y_{i}(t ; u)-z_{i}(t)\right), \quad t \in T, \quad \psi_{i}(\vartheta)=0, \quad i=1, \ldots, p
$$

By the Riesz theorem, the gradient $\nabla_{u} J_{1}^{(p)}(z, u) \in H$ at the point $u \in H$ is defined by the equality

$$
\nabla_{u} J_{1}^{(p)}(z, u)=b_{u}^{(p)} \in H, \quad \eta_{u}(h)=\left\langle b_{p}(\psi), h\right\rangle_{E}=\left\langle b_{u}^{(p)}, h\right\rangle_{H} \quad \forall h \in H
$$

In addition, under the specified conditions on the initial data of the problem, the derivative $J_{1 u}^{(p) \prime}(z, \cdot)$ and gradient $\nabla_{u} J_{1}^{(p)}(z, \cdot)$ satisfy the Lipschitz condition on $U_{H}$; i.e., there exists a number $L^{(p)} \geq 0$ such that, for any $u \in U_{H}$ and $v \in U_{H}$, the following inequalities hold:

$$
\begin{gathered}
\left\|J_{1 u}^{(p) \prime}(z, u)-J_{1 u}^{(p) \prime}(z, v)\right\|_{H^{*}} \leq L^{(p)}\|u-v\|_{H}, \\
\left\|\nabla_{u} J_{1}^{(p)}(z, u)-\nabla_{u} J_{1}^{(p)}(z, v)\right\|_{H} \leq L^{(p)}\|u-v\|_{H} .
\end{gathered}
$$

To construct minimizing sequences in problem (6.3), we apply the subgradient projection method

$$
u^{(k+1)}=\operatorname{Pr}\left(u^{(k)}-\beta_{k} v_{k}\right), \quad \beta_{k}>0, \quad v_{k} \in D_{u} F_{\alpha}^{(p)}\left(z, u^{(k)}\right), \quad k=0,1,2, \ldots,
$$

where $u^{(0)}$ is an initial approximation from $U_{H}, \operatorname{Pr}(w)$ is the projection in $H$ of the point $w \in H$ onto the set $U_{H}, D_{u} F_{\alpha}^{(p)}(z, u)$ is the set of subgradients of the subdifferential $\partial_{u} F_{\alpha}^{(p)}(z, u)$, and the parameters of the method $\beta_{k}$ should be chosen in an appropriate way.

Theorem 6.2. Let $u^{(0)}$ be an arbitrary initial approximation from $U_{H}$ in iterative process $(6.4)$, let $v_{k}$ be an arbitrary subgradient from $D_{u} F_{\alpha}^{(p)}\left(z, u^{(k)}\right)$, and let the parameters of the method $\beta_{k}$ satisfy conditions (5.12) and (5.13). Then, (1) $F_{\alpha}^{(p)}\left(z, u_{*}^{(k)}\right) \rightarrow \stackrel{\circ}{F_{\alpha}^{(p)}}(z)=\widetilde{F}_{\alpha}^{(p)}(z)$, where $F_{\alpha}^{(p)}\left(z, u_{*}^{(k)}\right)=\min \left\{F_{\alpha}^{(p)}\left(z, u^{(i)}\right): i \in \overline{0, k}\right\} ;(2)$ the minimizing sequence $\left\{u_{*}^{(k)}\right\} \subset U_{H}$ of problem (6.3) converges strongly in $E$ to the element $u_{\alpha}^{(p)}(z) ;(3) V\left[u_{*}^{(k)}\right] \rightarrow V\left[u_{\alpha}^{(p)}\right]$.

The proof of the theorem is similar to the proof of Theorem 5.1.

\section{NUMERICAL SIMULATION}

We perform the numerical simulation of the control reconstruction problem for the system

$$
\begin{gathered}
y_{t}=a^{2} y_{x x}, \quad(t, x) \in Q=T \times \Omega, \\
y(0, x)=y_{0}(x), \quad x \in \Omega=(0, l), \\
y(t, 0)=u(t), \quad y(t, l)=0, \quad t \in T=[0, \vartheta] .
\end{gathered}
$$

By the assumption, $y_{0} \in L_{2}(\Omega)$ and $a=$ const $>0$.

Let the set $P$ of geometric constraints on the controls be the interval

$$
P=\left[\mu_{1}, \mu_{2}\right] \subset \mathbb{R}, \quad \mu_{1}<\mu_{2} .
$$


Assume that the approximate measurement of the states of the dynamical system is simulated by the relation

$$
y_{\delta}(t, x)=y(t, x ; u)+\delta \xi(t, x), \quad \int_{T}\|\xi(t, \cdot)\|_{L_{2}(\Omega)}^{2} d t \leq 1 .
$$

In this specific case,

$$
\begin{gathered}
\lambda_{i}=\left(\frac{\pi i}{l}\right)^{2}, \quad \omega_{i}(x)=\sqrt{\frac{2}{l}} \sin \left(\sqrt{\lambda_{i}} x\right), \quad g^{(i)}=a^{2} \sqrt{\frac{2 \lambda_{i}}{l}}, \quad i=1,2,3, \ldots, \\
\dot{y}_{i}(t)=-a^{2} \lambda_{i} y_{i}(t)+g^{(i)} u(t), \quad t \in T, \quad y_{i}(0)=y_{0 i}, \\
y_{i}(t)=y_{i}(t ; u)=y_{0 i} \exp \left(-a^{2} \lambda_{i} t\right)+g^{(i)} \int_{0}^{t} \exp \left(-a^{2} \lambda_{i}(t-\tau)\right) u(\tau) d \tau, \\
y=y(t, x)=y(t, x ; u)=\sum_{i=1}^{\infty} y_{i}(t) \omega_{i}(x), \quad t \in T, \quad x \in[0, l] .
\end{gathered}
$$

Further, we fix some natural number $p \in \mathbb{N}$ and consider the system of ordinary differential equations

$$
\dot{y}_{i}(t)=-a^{2} \lambda_{i} y_{i}(t)+g^{(i)} u(t), \quad t \in T, \quad y_{i}(0)=y_{0 i}, \quad i=1, \ldots, p .
$$

Let us discretize problem (6.3). We fix a partition $\Delta$ of the interval $T$ by points $t_{k} \in T$, $0=t_{0}<t_{1}<\ldots<t_{m-1}<t_{m}=\vartheta$, with a uniform step $h$. On this partition $\Delta$, we define the discrete control $u_{h}=\left(u_{0}^{(h)}, \ldots, u_{m}^{(h)}\right)$ as an element of Euclidean space $\mathbb{R}^{m+1}$ satisfying the geometric constraint $u_{h} \in P^{m+1}=P_{h}$. The motion of system (7.1)

$$
y^{(p)}=y^{(p)}[t ; u]=\left(y_{1}(t ; u), \ldots, y_{p}(t ; u)\right), \quad t \in T,
$$

in the phase space $\mathbb{R}^{p}$ corresponding to the control $u \in U$ is approximated by the ordered set of grid functions

$$
y_{h}^{(p)}=\left(y_{1 h}, \ldots, y_{p h}\right), \quad y_{i h}=y_{i h}\left[\cdot ; u_{h}\right]=\left(y_{i h}\left[t_{0} ; u_{h}\right], \ldots, y_{i h}\left[t_{m} ; u_{h}\right]\right), \quad i=1, \ldots, p,
$$

and is called a discrete motion of system (7.1) in the phase space $\mathbb{R}^{p}$ corresponding to the discrete control $u_{h} \in P_{h}$. From (7.1), we find

$$
\begin{gathered}
y_{i h}\left[t_{k+1} ; u_{h}\right]=y_{i h}\left[t_{k} ; u_{h}\right] \exp \left(-a^{2} \lambda_{i} h\right)+g^{(i)} u_{k}^{(h)} a^{-2} \lambda_{i}\left(1-\exp \left(-a^{2} \lambda_{i} h\right)\right), \\
y_{i h}\left[t_{0} ; u_{h}\right]=y_{0 i}, \quad k=0, \ldots, m-1, \quad i=1, \ldots, p .
\end{gathered}
$$

The target functional in (6.3) is approximated by the finite sums

$$
F_{\alpha h}^{(p)}=F_{\alpha h}^{(p)}\left(z_{h}^{(p)}, u_{h}\right)=J_{1 h}^{(p)}\left(z_{h}^{(p)}, u_{h}\right)+\alpha J_{2 h}\left(u_{h}\right)+\alpha J_{3 h}\left(u_{h}\right)
$$

where the first two sums are the approximations by the trapezium method on the partition $\Delta$ of the integrals $J_{1}^{(p)}$ and $J_{2}(u)$, respectively, and the third sum $J_{3 h}$ is the approximation of the total variation $V[u]$ by the integral sum on the partition $\Delta$ in accordance with Lemma 5.1. 
To original infinite-dimensional extremal problem (6.3), we assign the finite-dimensional extremal problem

$$
\Phi_{\alpha h}^{(p)}\left(z_{h}^{(p)}\right)=\min \left\{F_{\alpha h}^{(p)}\left(z_{h}^{(p)}, u_{h}\right): u_{h} \in P_{h}\right\} .
$$

The solution to problem (7.2) is taken as a discrete approximation of the solution to problem (6.3). Problem (7.2) is uniquely solvable, since the target function $F_{\alpha h}^{(p)}\left(z_{h}^{(p)}, \cdot\right)$ is continuous and strictly convex on $\mathbb{R}^{m+1}$ and the set $P_{h}$ is compact in $\mathbb{R}^{m+1}$. The first two additive terms $J_{1 h}^{(p)}\left(z_{h}^{(p)}, \cdot\right)$ and $J_{2 h}(\cdot)$ of the function $F_{\alpha h}^{(p)}\left(z_{h}^{(p)}, \cdot\right)$ are continuously differentiable on $\mathbb{R}^{m+1}$, and the third additive term $J_{3 h}(\cdot)$ is not differentiable at all points $\mathbb{R}^{m+1}$ but is continuous and convex on $\mathbb{R}^{m+1}$ and, hence, is subdifferentiable on $\mathbb{R}^{m+1}$. Thus, the function $F_{\alpha h}^{(p)}\left(z_{h}^{(p)}, \cdot\right)$ is subdifferentiable on $\mathbb{R}^{m+1}$. We find an approximate solution to problem (7.2) using the subgradient projection method in the form

$$
u_{h}^{[k+1]}=\operatorname{Pr}_{h}\left(u_{h}^{[k]}-\beta_{k} v_{h}^{[k]}\right), \quad k=0,1, \ldots,
$$

where $D_{u} F_{\alpha h}^{(p)}\left(z_{h}^{(p)}, u_{h}^{[k]}\right) \ni v_{h}^{[k]}$ is a subgradient of the target function with respect to the variable $u_{h}$ at the point $u_{h}^{[k]}, \operatorname{Pr}_{h}\left(w_{h}\right)$ is the projection of the point $w_{h} \in \mathbb{R}^{m+1}$ onto the convex compact set $P_{h}$, some element $u_{h}^{[0]} \in P_{h}$ is chosen as an initial approximation, and $\beta_{k}$ is a sequence of parameters of the method satisfying conditions of form (5.12) and (5.13).

The set of subgradients $D_{u} F_{\alpha h}^{(p)}\left(z_{h}^{(p)}, u_{h}\right)$ of the target function is calculated rather simply, see [32]. Let us write only the subdifferential of the functional $J_{3 h}\left(u_{h}\right)$

$$
\partial J_{3 h}\left(u_{h}\right)=\partial\left[\sum_{k=0}^{m-1}\left|u_{k+1}^{(h)}-u_{k}^{(h)}\right|\right]=\sum_{k=0}^{m-1} d_{k}
$$

where $d_{k}=v_{k}$ for $u_{k+1}^{(h)}>u_{k}^{(h)}, d_{k}=-v_{k}$ for $u_{k+1}^{(h)}<u_{k}^{(h)}$, and $d_{k}=c o\left\{v_{k},-v_{k}\right\}$ for $u_{k+1}^{(h)}=u_{k}^{(h)}$. Here, $v_{k}=\left(0, \ldots, 0,-1_{k}, 1_{k+1}, 0, \ldots, 0\right)$ is the element of the space $\mathbb{R}^{m+1}$ that has -1 at the place numbered $k$ and 1 at the place numbered $k+1 ; \operatorname{co}\left\{v_{k},-v_{k}\right\}$ is the closed convex hull in $\mathbb{R}^{m+1}$ of two elements $v_{k}$ and $-v_{k}$.

The projection $\operatorname{Pr}_{h}\left(v_{h}\right)=\left(w_{0}, \ldots, w_{m}\right) \in \mathbb{R}^{m+1}$ of the element $v_{h}=\left(v_{0}, \ldots, v_{m}\right) \in \mathbb{R}^{m+1}$ onto the convex compact set $P_{h}$ is calculated by the rule: $w_{k}=v_{k}$ for $v_{k} \in P, w_{k}=\mu_{1}$ for $v_{k}<\mu_{1}$, and $w_{k}=\mu_{2}$ for $v_{k}>\mu_{2}, k=0, \ldots, m$.

The measurement errors in the discrete problem are simulated by the relations

$$
\xi_{i h}(t)=\varkappa_{i} \sin \left(\nu_{i} t\right), t \in T, \nu_{i}=\text { const }_{i}, \varkappa_{i}=\text { const }_{i}, i=1, \ldots, p, \vartheta\left(\varkappa_{1}^{2}+\ldots+\varkappa_{p}^{2}\right) \leq 1 .
$$

The numerical experiments are performed for the following parameters of the problem: $a=1$, $\vartheta=1, l=1, \mu_{1}=-2, \mu_{2}=2, y_{0}=0, \nu_{i}=2, \varkappa_{i}=p^{-1}, i=1, \ldots, p$.

As the model controls to be reconstructed, we choose the functions:

(1) $u=u_{(1)}(t)=2 t \sin \left(40 t^{1.5}\right)$ (the smooth control);

(2) $u=u_{(2)}(t)=1.5$ for $0.2<t<0.25,-1$ for $0.4<t<0.43,-2$ for $0.6<t<0.62,2$ for $0.62 \leq t<0.63$, and 0 at the remaining points of the interval $[0,1]$ (the impulse control).

The values $\gamma_{k}$ are chosen by the formulas $\gamma_{0}=1, \gamma_{k}=1 / \sqrt{k}, k=1,2, \ldots$ The initial function in the subgradient projection method is the zero grid function. It is rather far from the model control in both the norm and qualitative behavior. 




Fig. 1. Reconstructing the smooth control.

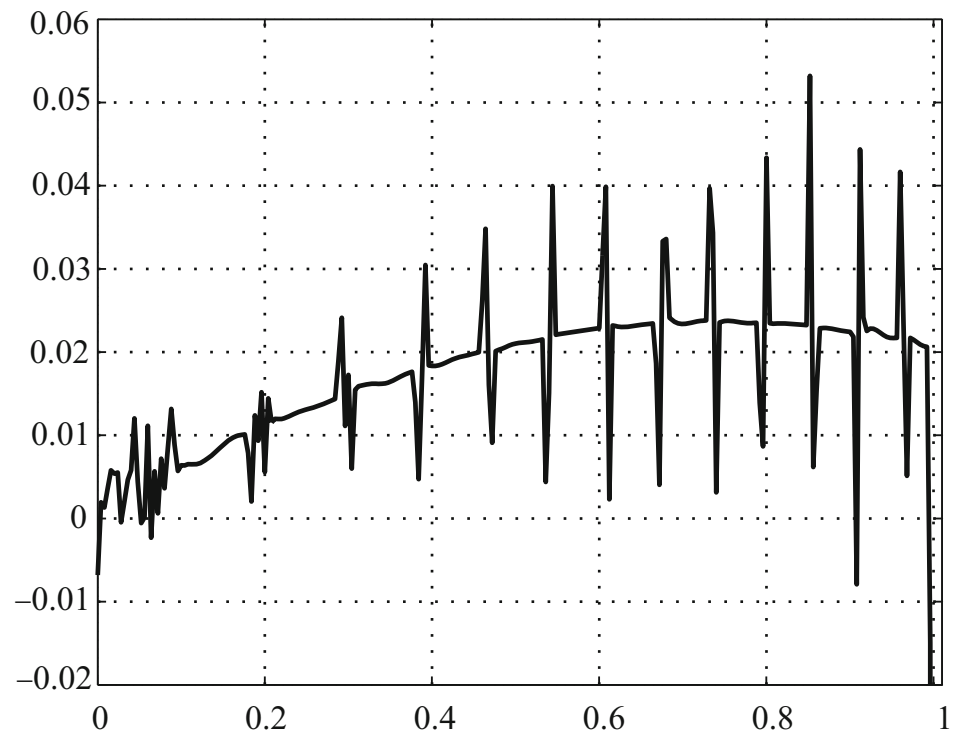

Fig. 2. Error of reconstructing the smooth control.

Table 1. Reconstructing the smooth control

\begin{tabular}{|c|c|c|}
\hline Error $\delta$ & Residual & Relative error \\
\hline 3.0 & 0.11626 & 14.41770 \\
2.0 & 0.07901 & 9.79809 \\
1.0 & 0.04200 & 5.33222 \\
0.5 & 0.02699 & 3.34666 \\
0.2 & 0.02041 & 2.53091 \\
\hline
\end{tabular}






Fig. 3. Reconstructing the impulse control.

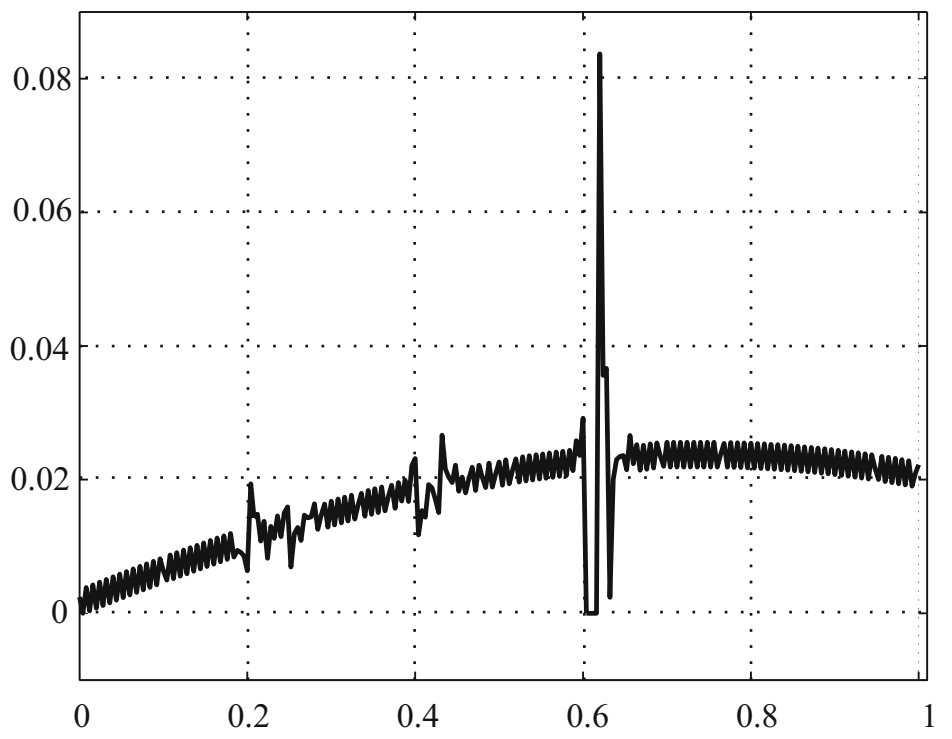

Fig. 4. Error of reconstructing the impulse control.

Table 2. Reconstructing the impulse control

\begin{tabular}{|c|c|c|}
\hline Error $\delta$ & Residual & Relative error \\
\hline 3.0 & 0.11656 & 24.19850 \\
2.0 & 0.07772 & 16.13540 \\
1.0 & 0.03888 & 8.07293 \\
0.5 & 0.01950 & 4.04921 \\
0.2 & 0.00793 & 1.64574 \\
\hline
\end{tabular}


The dependence of the parameter $\varepsilon=\varepsilon(\delta)$ of minimization accuracy in problem (7.2) on the error $\delta$ of measuring the states of the dynamical system is not controlled directly; the accuracy of the solution to problem (7.2) is determined by choosing the number of iterations $M$ in the subgradient projection method and the value of the step $h$ characterizing the degree of problem discretization.

In Figures 1-4, we present the calculation results for the following values of parameters: $h=$ $0.004, M=500, \alpha=10^{-4} \delta$, and $N=15$.

In Figures 1 and 3, we show the model controls to be reconstructed by the solid line, the reconstruction result for $\delta=3$ by the dashed line, and the reconstruction result for $\delta=0.5$ by the dotted line. The reconstruction accuracies for $\delta=0.5$ are presented in Figures 2 and 4 (the plots for the difference between the model control and its reconstruction are shown). In these figures, the horizontal axis reflects time and the vertical axis reflects the control and reconstruction accuracy values, respectively.

The results of reconstructing the control for varying parameter $\delta$ are presented in Tables 1 and 2 ; for the relative error, the percentage is given.

\section{ACKNOWLEDGMENTS}

This work was supported by the Ural Branch of the Russian Academy of Sciences (project no. 09P-1-1009) within the Program for Fundamental Research of the Presidium of the Russian Academy of Sciences "Fundamental Problems of Nonlinear Dynamics" and by the Russian Foundation for Basic Research (project no. 11-01-00073).

\section{REFERENCES}

1. L. S. Pontryagin, V. G. Boltyanskii, R. V. Gamkrelidze, and E. F. Mishchenko, The Mathematical Theory of Optimal Processes (Fizmatgiz, Moscow, 1961; Wiley, New York, 1962).

2. N. N. Krasovskii, The Theory of Motion Control (Nauka, Moscow, 1968) [in Russian].

3. N. N. Krasovskii and A. I. Subbotin, Positional Differential Games (Nauka, Moscow, 1974) [in Russian].

4. Yu. S. Osipov and A. V. Kryazhimskii, Inverse Problems for Ordinary Differential Equations: Dynamical Solutions (Gordon and Breach, London, 1995).

5. Yu. S. Osipov, F. P. Vasil'ev, and M. M. Potapov, Foundations of the Dynamical Regularization Method (Mosk. Gos. Univ., Moscow, 1999) [in Russian].

6. A. B. Kurzhanskii, Control and Observation under Uncertainty (Nauka, Moscow, 1977) [in Russian].

7. F. L. Chernous'ko and A. A. Melikyan, Game Problems of Control and Search (Nauka, Moscow, 1978) [in Russian].

8. V. I. Maksimov, Dynamical Inverse Problems of Distributed Systems (Izd. UrO RAN, Yekaterinburg, 2000; VSP, Utrecht-Boston, 2002).

9. P. D. Krut'ko, Inverse Problems of the Dynamics of Control Systems: Nonlinear Models (Nauka, Moscow, 1988) [in Russian].

10. A. N. Tikhonov and V. Ya. Arsenin, Methods for Solving Ill-Posed Problems (Nauka, Moscow, 1979) [in Russian].

11. V. K. Ivanov, V. V. Vasin, and V. P. Tanana, Theory of Linear Ill-Posed Problems and Its Applications (Nauka, Moscow, 1978; VSP, Utrecht, 2002).

12. M. M. Lavrent'ev, V. G. Romanov, and S. P. Shishatskii, Ill-Posed Problems of Mathematical Physics (Nauka, Moscow, 1980; Amer. Math. Soc., Providence, RI, 1986).

13. A. L. Ageev, Zh. Vychisl. Mat. Mat. Fiz. 20 (4), 819 (1980).

14. V. V. Vasin, Doklady Math. 63 (1), 5 (2001).

15. V. V. Vasin, Doklady Math. 71 (3), 419 (2005). 
16. V. V. Vasin, Proc. Steklov Inst. Math., Suppl. 1, S247 (2006).

17. V. V. Vasin and M. A. Korotkii, J. Inverse Ill-Posed Probl. 15 (8), 853 (2007).

18. V. V. Vasin and T. I. Serezhnikova, Russian Math. (Iz. VUZ) 45 (4), 1 (2001).

19. V. V. Vasin and T. I. Serezhnikova, Autom. Remote Control 65 (2), 270 (2004).

20. A. N. Tikhonov, A. S. Leonov, and A. G. Yagola, Nonlinear Ill-Posed Problems (Nauka, Moscow, 1995; Chapman and Hall, London, 1998).

21. A. S. Leonov, J. Inverse Ill-Posed Probl. 13 (6), 595 (2005).

22. A. S. Leonov, Zh. Vychisl. Mat. Mat. Fiz. 22 (3), 516 (1982).

23. A. S. Leonov, Solution of Ill-Posed Inverse Problems: Study of Theory, Practical Algorithms, and Demonstrations in MATLAB (Librokom, Moscow, 2010) [in Russian].

24. E. Giusti, Minimal Surfaces and Functions of Bounded Variation (Birkhäuser, Basel, 1984).

25. R. Acar and C. R. Vogel, Inverse Problems 10, 1217 (1994).

26. G. Chavent and K. Kunish, ESAIM Control Optim. Calc. Var. 2, 359 (1997).

27. C. R. Vogel, Computation Methods for Inverse Problems (SIAM, Philadelphia, 2002).

28. M. A. Korotkii, Russian Math. 53 (2), 68 (2009).

29. M. A. Korotkii, J. Appl. Math. Mech. 73 (1), 26 (2009).

30. M. A. Korotkii, Candidate's Dissertation in Physics and Mathematics (Yekaterinburg, 2009).

31. D. O. Soboleva, Vestn. Buryatsk. Gos. Univ., Ser. Mat. Informatika 9, 59 (2010).

32. A. I. Korotkii and D. O. Mikhailova, Trudy Inst. Mat. Mekh. UrO RAN 16 (4), 211 (2010).

33. A. I. Korotkii and E. I. Gribanova, Proc. Steklov Inst. Math. 275, Suppl. 1, S68 (2011).

34. O. A. Ladyzhenskaya, The Boundary Value Problems of Mathematical Physics (Nauka, Moscow, 1973; SpringerVerlag, Berlin, 1985).

35. O. A. Ladyzhenskaya, V. A. Solonnikov, and N. N. Ural'tseva, Linear and Quasilinear Equations of Parabolic Type (Nauka, Moscow, 1967; Amer. Math. Soc., Providence, 1968).

36. O. A. Ladyzhenskaya and N. N. Ural'tseva, Linear and Quasilinear Elliptic Equations (Nauka, Moscow, 1973; Academic, New York, 1968).

37. J.-L. Lions, Optimal Control of Systems Governed by Partial Differential Equations (Springer-Verlag, Berlin, 1971; Mir, Moscow, 1972).

38. A. I. Korotkii, Doctoral Dissertation in Physics and Mathematics (Yekaterinburg, 1993).

39. F. P. Vasil'ev, Optimization Methods (Faktorial, Moscow, 2002) [in Russian].

40. A. D. Ioffe and V. M. Tikhomirov, Theory of Extremal Problems (Nauka, Moscow, 1974) [in Russian].

41. B. T. Polyak, Introduction to Optimization (Nauka, Moscow, 1983; Optimization Software, New York, 1987).

42. V. F. Dem'yanov and L. V. Vasil'ev, Nondifferentiable Optimization (Nauka, Moscow, 1981; Springer-Verlag, New York, 1985).

43. A. N. Kolmogorov and S. V. Fomin, Elements of the Theory of Functions and Functional Analysis (Nauka, Moscow, 1972; Dover, NewYork, 1999).

44. L. A. Lyusternik and V. I. Sobolev, Elements of Functional Analysis (Nauka, Moscow, 1965) [in Russian].

45. I. P. Natanson, Theory of Functions of a Real Variable (Nauka, Moscow, 1941; Ungar, New York, 1955) [in Russian].

Translated by V. Rozenberg 